\title{
Multiresonator imaging sensor for the aerial parameters detection
}

\author{
Anton V. Saetchnikov, Elina A. Tcherniavskaia, Vladimir A. Saetchnikov and Andreas Ostendorf
}

\begin{abstract}
Applicability of the whispering gallery mode sensor in the form of the multiresonator imaging device for the atmosphericrelevant parameters monitoring has been demonstrated. The sensor design and the appropriate techniques for sensing data collection have been discussed. The sensor performance has been numerically and experimentally studied on examples of the temperature variation detection and the monitoring of small spherical microparticles $(<3 \mu \mathrm{m})$ that have been used to mimic the particulate contamination of the environment. The thermal sensitivity of the sensor on the level of $8.6 \mathrm{pm} /{ }^{\circ} \mathrm{C}$ has been experimentally demonstrated. The experimental results on the distinguishing of the microparticle concentrations $(0.5 \%$ and $1 \%)$ via the wavelength sweeping technique and the results on the single particle detection under illumination at the fixed wavelength have been shown.
\end{abstract}

Keywords-microresonators, whispering gallery mode, multiplexing, temperature sensing, particulates detection

\section{INTRODUCTION}

The optical sensing techniques employing the laser light as a probe belong to the most efficient approaches for characterization of the changes in the physical and chemical parameters of the object under study. Compared to the conventional electrical and electronic sensors, the optical devices benefit from the immunity to the electromagnetic (EM) interference, enable multiparameter quantification (e.g., temperature, pressure, and refractive index at the same time), lightness, external perturbation nature as well as the distributed configurations. Despite the broad palette of the compact, fast, and highly sensitive optical solutions developed in the last decades, the possible range for their application as the onboard sensors is still mainly restricted to the imaging tasks and the laser-assisted distance measurements.

One of the most intensively researched optical sensing techniques arose from the resonance phenomenon of the whispering gallery modes (WGM) occuring in the microcavities [1]-[3]. The WGMs are formed in an optical ring resonator where the light is guided along the resonator's periphery due to the multiple total internal reflections so that the returning wave starts to interfere with itself. The field of the WGM is dominantly located within the microcavity and partly penetrates via the evanescent field into the external environment. The mechanism for the detection of the external parameter variations is based on the monitoring of the WGM spectral changes: the resonance frequency shift, linewidth broadening, and mode splitting [4]. For this, several shapes of the microresonators have been proposed: a sphere, a cylinder, a disk, and a toroid [5]-[9]. The materials for the cavities fabrication include glass, polymers, quartz, etc.

The main benefit of the optical microresonators - the high spectral resolution and thus the low detection limit - is determined by the high quality (Q-) factor of the cavity which exceeds for particular realizations $10^{10}$ in the air [10]. Here the Q-factor is not only determined by the inherent properties of the microresonator but also by the probing configuration. Therefore, the free space excitation and the remote monitoring of the WGMs is mostly avoided due to the low efficiency and the necessity for the near-evanescent light coupling mechanisms (e.g. via an optical prism, cut-edged, or tapered optical fiber). Alternatively, the remote WGM excitation may be supported by the doping of the microresonator material with the fluorescent components to reach the lasing conditions [11], [12]. This, however, requires an external cumbersome spectroscopic unit for signal collection suffering from the limited resolution.

The widely used tapered optical fiber mechanism guarantees up to $>99 \%$ coupling efficiency. At the same time, it is highly influenced by the external vibrations, airflow disturbances, change of the sensed medium, and environmental dust that impact the coupling conditions and thus the measurement accuracy. Several solutions to fix the position of the excitation fiber with respect to the resonator via the UV curable polymer spot [13] or packaging of the fiber together with the microcavity in a post-solidified polymer matrix [14] have been proposed to address the robustness issue. The latter has been recently verified on the capability of the approach for integration within an airborne device [15] for the temperature measurements. Another option to be utilized as an aerial sensor may be associated with the fabrication of a planar ring resonator together with a waveguide on a single substrate [16], [17]. However, the planar resonators possess a lower Q-factor than the toroid or sphere microresonators. Alternatively, the recently proposed microresonator-based insturument [18] in the form of the simultaneously interrogated multiple low-cost spherical glass microresonators and tested for small molecules adsorption and refractive index variations detection might act as a trade-off option between the fabrication complexity, sensitivity, robustness, and affordability.

In this paper we report on the applicability of the multiple optical microresonator-based imaging sensor for the aerial parameter detection and study both numerically and experimentally the sensor response on the temperature variations and on the particulate contamination by utilizing the wavelength sweeping and the fixed frequency signal collection methods.

\section{MAterials AND MethodS}

\section{A. Sensor design}

A silica microsphere ( mean diameter of $100 \mu \mathrm{m}$, Cospheric LLC) acts as an individual WGM microresonator among many 
others on the chip. In order to reduce the light scattering (losses) and enhance the resonator Q-factor, each microsphere is ultrasonically processed under the methanol for 10 min with the subsequent acetone flushing and then drying procedure in the oven at $95^{\circ} \mathrm{C}$.

The stable coupling conditions for the optical microcavities are guaranteed by the deposition of a thin film of a low refractive index polymer (MY-133MC) on a right angle optical prism by the spin coating procedure. This adhesive solution is chosen due to its excellent stability in the aqueous environment. The microresonators are then placed onto the surface of the adhesive layer via the free fall, whereupon the sensor is dried for the polymer film solidification. Next, the assembly is gently flushed with the isopropanol to remove both possible contaminating particles and non-fixed microspheres. Finally, the microcavities are enclosed with a PMMA housing which contains a glass window and the input/output channels. Hereby, a measurement cell is formed around the microcavities that imitates the environmental chamber ( Fig. 1). In order to monitor the thermal variations inside the cell a temperature probe (PT1000) has been put in contact with its inner environment and fixed within the cover.

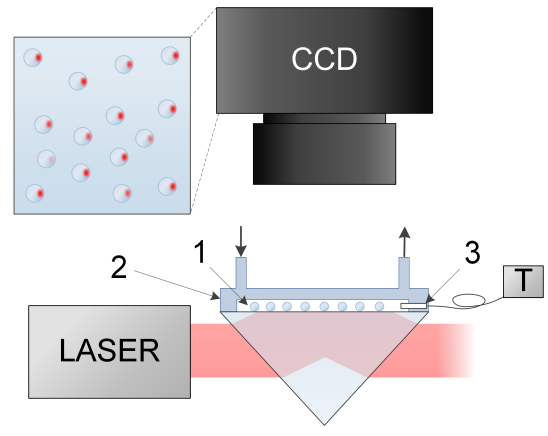

Fig. 1. Experimental setup design: (1) measurement chamber with microresonators, (2) PMMA cover, (3) temperature probe.

Excitation of the WGMs in the microcavities is performed with the tunable diode laser (New Focus, $680 \mathrm{~nm}$ ) with the linewidth of $200 \mathrm{kHz}$. The lasing source has been operated in two regimes: 1) the laser wavelength tuning which enables the WGM spectrum observation and 2) at a constant wavelength. The signal has been collected in the imaging scheme consisting of an objective and a monochrome CCD camera (VRMagic vmrc 3+). This interrogation approach empowers the parallel signal acquisition from multiple microcavities, whereas the temporal and spectral resolution is determined by the camera frame rate and/or the laser wavelength tuning speed, respectively. The selected configuration of the imaging system magnification and the camera resolution allow concurrent observation of up to $\sim 300$ microspheres.

The method of the resonator fixation ensures the robustness of the light coupling conditions during the sensing event i.e. their weak susceptibility to the external factors. In addition, the light coupling is enabled hereby into all microresonators at once. At the same time, the dispersion in the arrangement of the microcavities, variation of their number among the sensor replicates as well as deviation of their performance provide a unique spectral response for each device. The proposed sensor configuration tolerates the failure of the resonance conditions for some of the microresonators. The latter may be observed due to the variant coupling and/or scattering losses mainly dependent on the physical properties of the individual microsphere, and is compensated by the abundance (up to several hundreds) of the microresonators allocated on the sensor.

\section{B. Sensor characterization}

Due to some randomness in the actual number of the microresonators on the sensor, their position, and deviation of their sensing efficiency, the device characterization is performed prior to the measurements in order to ensure the reproducibility of the sensing results. This includes 1) the localization of the microcavities on the sensor and 2) the association of the particular light out-coupling areas with the corresponding microspheres [19].

The method for the microspheres localization implies several steps: 1) image enhancement regulated by denoising, sharpening, background correction, and histogram equalization procedures; and 2) resonator edges detection realized by the circular Hough transform. Analysis of the proposed algorithm performance on the variety of the sensor samples shows its high sensitivity to the spherical shape of the sensing units, allows successful allocation of $>95 \%$ of the individual microresonators and excludes the dust particles and/or corrupted microresonators (typically of the non-spherical shape) out of consideration (Fig. 2a). The microcavities that do not exhibit a resonance behaviour are not included into the further analysis as well.

The high contrast between the WGM signal and the background illumination allows direct matching of the captured out-coupled light distribution to the previously defined microresonator areas. This, in turn, enables the collection of the WGM data within an acceptable for the real-time calculations time period. The collected WGM spectra for the whole set of the localized microresonators form a spectral map of the multicavity sensor which is shown in Fig. 2b. The common values for the loaded Q-factors of the microcavities lie in the range from $10^{3}$ to $10^{5}$. A representative WGM spectrum collected in the water environment is represented in Fig. 2c.

\section{Data processing}

Two different methods for the data collection, processing, and interpretation have been employed in the studies (Fig. 3). The first one, the laser wavelength sweeping technique, delivers a set of the spectral maps together with the corresponding time points. This data is processed by the sequential analysis of each resonator's spectrum and the respective spectral changes are characterized as described in detail in [21]. Initially, each WGM spectrum associated with an individual resonator is denoised by the stationary wavelet transform. The following peaks picking, fitting, and F-test allow to describe the raw data by a set of parameters (mode's frequency, linewidth, and peak intensity) with the mean squared error (MSE) below $5 \times 10^{-5}$. Finally, the dynamics of the resonance frequency shift for the 

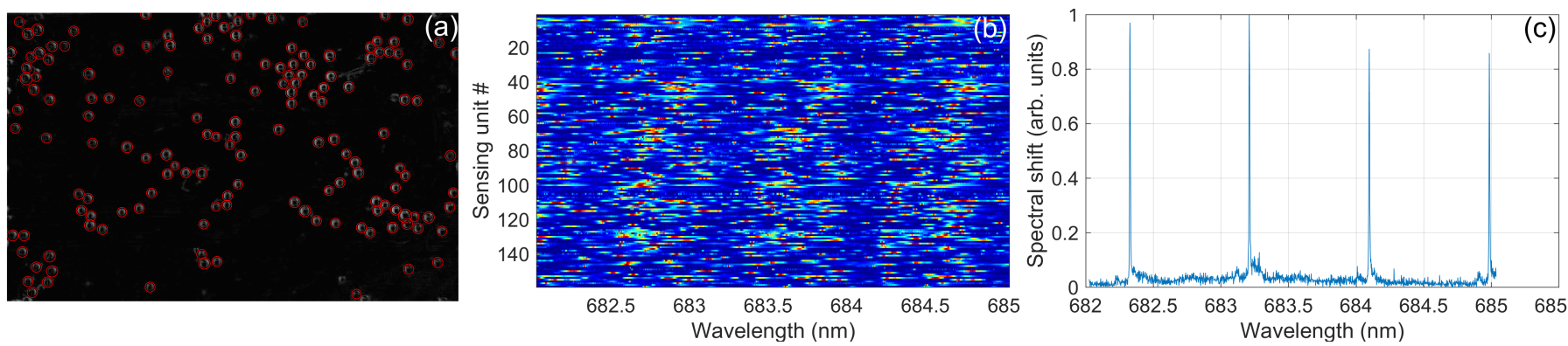

Fig. 2. Sensor design and characterization [20] (ㅇ2020] IEEE). (a) Overview of the multiresonator imaging sensor. Red edges show the results of the microresonator localization procedure. (b) Spectral map for the sensor. (c) A representative WGM spectrum for a single microresonator.

particular resonators as well as the common one for the sensor as a whole (calculated with the help of the principle component analysis (PCA) as described in [18]) are extracted.
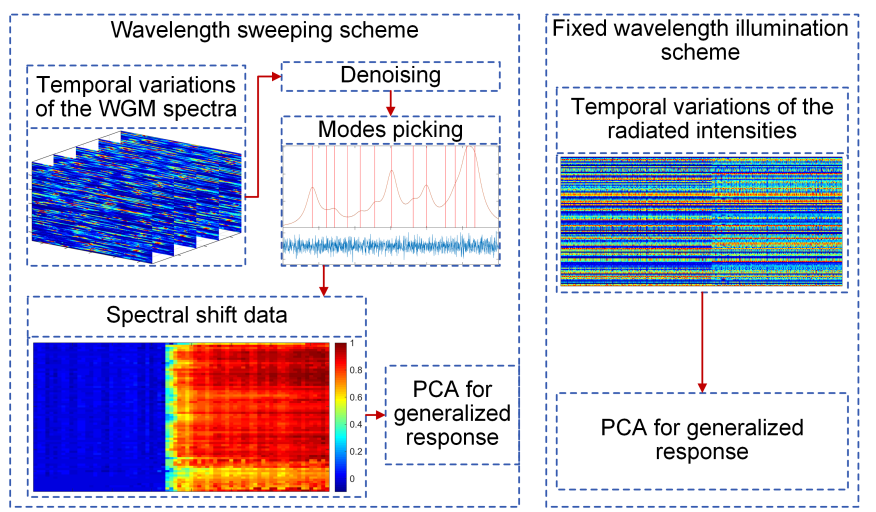

Fig. 3. Data processing flow charts for the wavelength sweeping and the fixed wavelength illumination schemes.

Another signal collection approach is based on the fixed wavelength illumination and generates a data set that represents the time-dependent change of the scattered by the microresonators light intensities. This variation represents a nonlinearly modulated response that is regulated by the spectral properties of the individual microresonators. PCA of this data, where the time points are considered as objects and the intensities of the sensing units as variables, allows to extract the generalized sensor reaction. It has been previously demostared [18] that for more than a hundred resonators in the scope the median value of the correlation coefficient between the variations retrieved with the first and the second interrogation approaches reaches 0.99 , where the laser wavelength selection for illumination has a minor impact on the sensing results.

\section{RESULTS}

The tasks for the temperature variation measurement and the particulate matter (PM) detection have been selected to demonstrate the applicability of the multiresonator imaging sensor for the aerial parameters inspection. Both numerical estimations (subsection A) and the experimental results (subsections $\mathrm{B}, \mathrm{C}$ ) on the sensor response on the external variations are analysed.)

\section{A. Numerical estimations}

According to the quasi-geometrical approximation the spectral position (wavelength $\lambda$ ) of each WGM with a polar mode number $(m)$ in the microresonator of radius $R$ follows the equation $\left(m \lambda=2 \pi R n_{e f f}\right)$ with $n_{e f f}$ standing for the effective refractive index. The variations of the thermodynamic conditions consist of the thermal expansion of the microresonator material and the change of $n_{\text {eff }}$. The spectral shift of the resonant wavelength induced due to these variations can be defined according to the following equation:

$$
\frac{1}{\lambda} \frac{d \lambda}{d T}=\frac{1}{R} \frac{d R}{d T}+\frac{1}{n_{e f f}} \frac{d n_{e f f}}{d T}
$$

with $T$ denoting the temperature and $n_{\text {eff }}$ derived as:

$$
n_{e f f}=\frac{1}{\int_{V_{o}+V_{i}}|E|^{2} d V}\left(n_{r} \int_{V_{i}}|E|^{2} d V+n_{m} \int_{V_{o}}|E|^{2} d V\right) .
$$

Here $E$ is the EM field amplitude, $V_{o}$ and $V_{i}$ stand for the outer (environment) and inner (resonator) volumes correspondingly, $n_{r}$ is the resonator refractive index, $n_{m}$ is the sensed medium refractive index.

In Eq. (1) the first term describes the thermal expansion coefficient whereas the second - the thermo-optic coefficient. Taking into account that both thermal expansion and thermooptic coefficients for the soda lime glass are equal to $9 \times$ $10^{-6} K^{-1}$, the expected temperature shift on $+1^{\circ} \mathrm{C}$ will result in a $12 \mathrm{pm}$ positive shift of the resonant frequency for the $685 \mathrm{~nm}$ wavelength. The given above estimations are valid for the case when the WGM is fully located in the cavity and thus represents the upper sensitivity limit.

For the case of the PM detection the sensor response can appear in the following forms:

a) Reaction on the single/multiple particles that interact with the EM field penetrating into the external medium: In contrast to the nanoparticle or molecular sensing, where the common sensing mechanism is described by the adsorption or coupling to the receptor, the mentioned process is not a driving factor for the PM sensing primary due to the size of the particles in the range of several micrometers. Here, a short-term reaction of the sensor on the particle located in the vicinity to the microcavity surface is dominating. The extent of this reaction is characterized by the optical properties of the particle, its size, and distance to the resonator surface. 

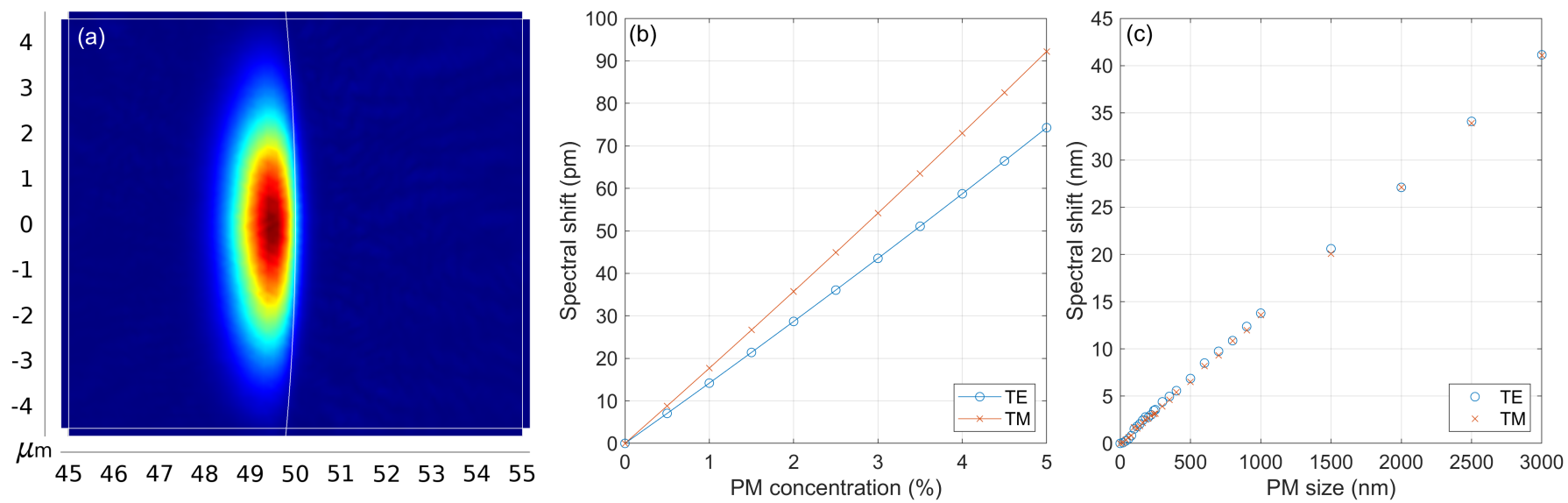

Fig. 4. Numerical results on the simulation of the WGM sensor response on the microparticle suspension. (a) EM field distribution for the fundamental ( $\mathrm{m}=$ 684) TE mode. (b) Numerical estimations on the spectral shift of the fundamental mode frequency with the change of the PS microparticle mass concentration. (c) Numerical estimations on the spectral shift of the fundamental mode frequency with the change of the PS microparticle size deposited as a homogeneous layer.
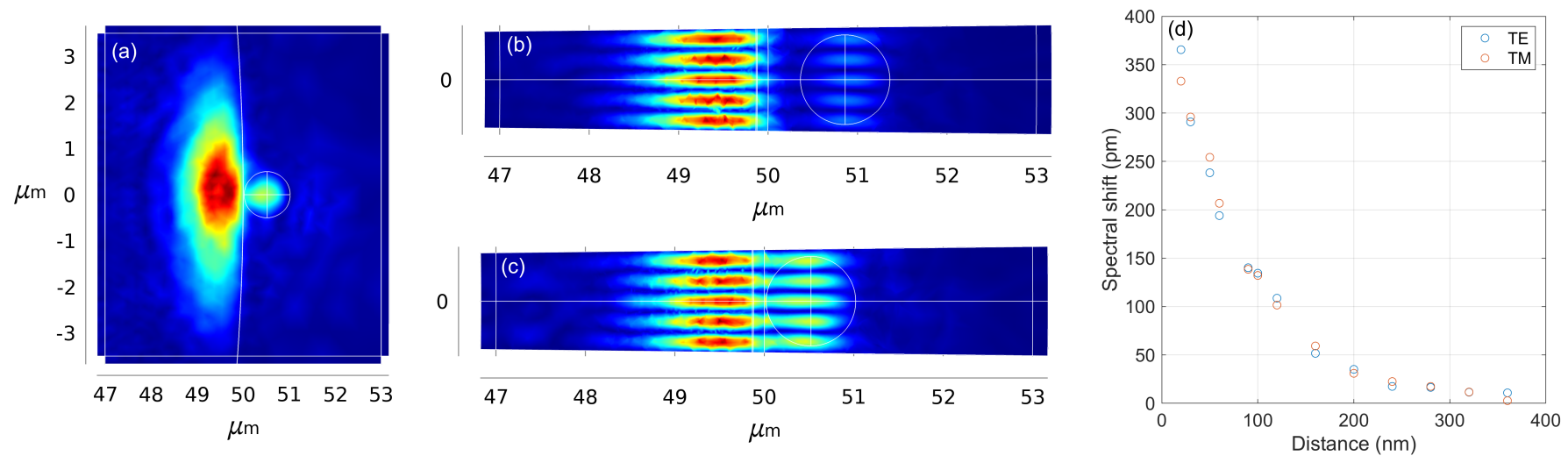

Fig. 5. Numerical results on the simulation of the WGM sensor response on a single PS microparticle (mesh element size limited by the computational resources causes the moderate image resolution). (a) EM field distribution for the microresonator transversal cross-section. EM field distribution for the microresonator longitudinal cross-section when the microparticle is located at $360 \mathrm{~nm}$ (b) and at $20 \mathrm{~nm}$ (c) to the surface. (d) Numerical estimations on the spectral shift of the fundamental mode frequency with the change of the position of the PS microparticle relative to the resonator surface.

b) The particle concentration influence on the frequency of the sensor response: Another type of the sensor reaction implies spectral changes caused by the variations of the bulk refractive index due to the particles distributed in the environment.

In general, both sensor reactions are observed simultaneously.

In order to check the capabilities of the WGM sensor for the PM detection, we performed numerical simulations using the finite element method for searching for the eigenfrequencies in the Comsol software. A 3D model of $\lambda / 2$ cross-section of a microresonator with the reflective boundaries for the section faces that emulate the standing wave formation has been selected to simulate a single PM component detection. In order to avoid the undesirable reflections from the boundaries of the computational domain, we introduced the perfectly matched layers (PML) with $\lambda / 4$ thickness at the boundaries of the simulation region. The eigenfrequencies have been searched in the vicinity to the laser wavelength tuning range of [680:690] $\mathrm{nm}$. An example of the EM field distribution for the fundamental TE mode for the current configuration is represented in Fig. 4a.

The PM detection principle has been emulated by the polysterene (PS) microspheres of several volumetric concentrations and several sizes. The dependence of the calculated value of the resonance wavelength for the fundamental mode of a $100 \mu \mathrm{m}$ glass microresonator $(\mathrm{n}=1.519)$ near the wavelength of $685 \mathrm{~nm}$ on the volumetric concentration $(c)$ of the PS microparticles $\left(n_{p}=1.583\right)$ in the aqueous environment $\left(n_{m}=1.33\right)$ is shown in Fig. 4b. The resulting refractive index of the microparticle suspension $\left(n_{s}\right)$ has been derived as $n_{s}=(1-c) \cdot n_{m}+c \cdot n_{p}$. The results show a linear behaviour of the spectral shift on the particle concentration changes where the growth factor reaches $14.8 \mathrm{pm} / \%$ for the TE mode and $18.4 \mathrm{pm} / \%$ for the TM mode.

The simulation results on the possible deposition of a homogeneous layer of PS microparticles with the size up to $3 \mu \mathrm{m}$ onto the microresonator surface is represented in Fig. 4c. The results show a linear behaviour of the spectral shift versus the thickness of the deposited layer with the slope of $\approx 13 \mathrm{~nm}$ per $1 \mu \mathrm{m}$ of the thickness increase. PM2.5 particles 

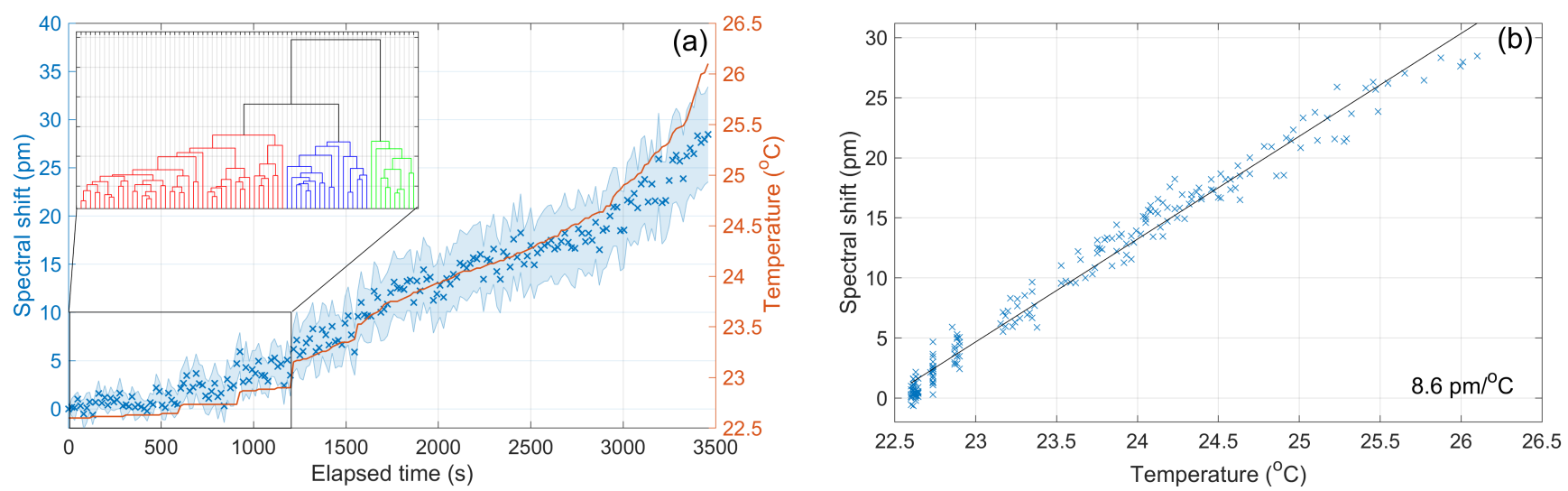

Fig. 6. Temperature variation detection with the multiresonator imaging sensor [20] (@[2020] IEEE). (a) Sensor temporal response on the temperature variations generalized over individual microresonators (shaded area shows the 95\% confidence interval). The inset represents a dendrogram for the generalized sensor response at slightly varying temperature conditions. (b) Dependence of the mean spectral shift on the temperature changes.

layer formation is expected to result in the spectral shift up to $35 \mathrm{~nm}$ which significantly exceeds the free spectral range value $(1 \mathrm{~nm})$.

In order to numerically estimate the impact of a single PS microparticle on the spectral properties of the WGM mode the simulation region has been extended to $5 \cdot \lambda / 2$ section of the resonator (the size of the simulation domain is limited by the available computational resources). Overview of the calculated EM distribution of the fundamental mode in this simulation geometry is represented in Fig. 5a-c. The induced spectral shift for the fundamental mode by the presence of a single $1 \mu \mathrm{m}$ PS particle at the varied distance from 20 to $360 \mathrm{~nm}$ is represented in Fig. 5d. Localization of the PS microparticle in the close vicinity to the sensing area induces the spectral shift for the fundamental mode on $\approx 350 \mathrm{pm}$. Following the the exponential decay the evanescent field exhibits in the environment, the spectral shift governed by the distance of the microparticle to the resonator surface drops exponentially as well. It sinks down to $10 \mathrm{pm}$ when a single microparticle moves away from the microresonator surface on $360 \mathrm{~nm}$.

\section{B. Temperature variations sensing}

The performance of the discussed microresonator based imaging sensor for the temperature variations detection has been tested in the experiment where the thermal changes are generated by the heating element (IKA RCT basic) located in the vicinity to the sensor. The heat has been transferred to the sensing chamber via the water flow in order to ensure the controllable thermal conditions and equal heat distribution over the multiple microresonators.

The temperature within the measurement cell has been varied from $22.6^{\circ} \mathrm{C}$ to $26.1^{\circ} \mathrm{C}$. The spectral shift change for the microcavities and measured with the wavelength sweeping technique is represented in Fig. 6a. It has been determined that the temporal variations of the temperature match the observed dynamics of the spectral shift. The temperature increase on $3.5^{\circ} \mathrm{C}$ is followed by the positive spectral shift of the resonance wavelength on $28 \mathrm{pm}$. The variation between the sensing properties of the single microresonators appears in the form of the enhanced deviation of the spectral shift value up to $5 \mathrm{pm}$.

In order to check whether the slight temperature variations at the initial time points can be distinguished, we performed the hierarchical clustering. The time points are treated here as the objects each characterized by a set of spectral shift parameters of all sensing units. The Chebyshev distance measure and average linkage have been selected to form a cluster tree. The dendrogram representing the hierarchical cluster structure shows a clear separation of the data into three clusters. Each of them consists of exactly the time points affiliated with a certain temperature level and thus the observed results can be clearly distinguished.

Variation of the spectral shift as a function of the temperature is demonstrated in Fig. 6b. The spectral shift values accurately (MSE $<10^{-3}$ ) follow the linear function and are characterized by the mean sensitivity of $8.6 \mathrm{pm} /{ }^{\circ} \mathrm{C}$. This value is close to the previously estimated numerical result $\left(12 \mathrm{pm} /{ }^{\circ} \mathrm{C}\right)$ where the slight disparity is caused by the partial localization of the EM field outside the microcavity.

\section{Microparticles detection}

The performance of the PM detection with the WGM sensor has been analysed in the aqueous environment where the homogeneous particle flow over the sensing area has been ensured via a peristaltic pump. Several suspensions of the PS particles have been used in the current study: $3 \mu \mathrm{m}$ particles (Sigma Aldrich, 79166-5ml-f) have been selected to mimic the PM10; $0.9 \mu \mathrm{m}$ ones (Microparticles GmbH, PS-F-0.9) were employed to simulate the detection of PM2.5.

The experimental results on the temporal dynamics of the spectral shift changes for $0.5 \%$ suspension of the $0.9 \mu \mathrm{m}$ microparticles and $1 \%$ suspension of the $3 \mu \mathrm{m}$ microparticles are represented in Fig. 7a. The saturation value for the spectral shift at $1200 \mathrm{~s}$ after the suspension injection reaches $8 \mathrm{pm}$ for the PM2.5-like particles and $16 \mathrm{pm}$ for the PM10-like particles. Differences between the microresonator characteristics cause the deviation in the output values among the individual microcavities which increases for both suspensions 

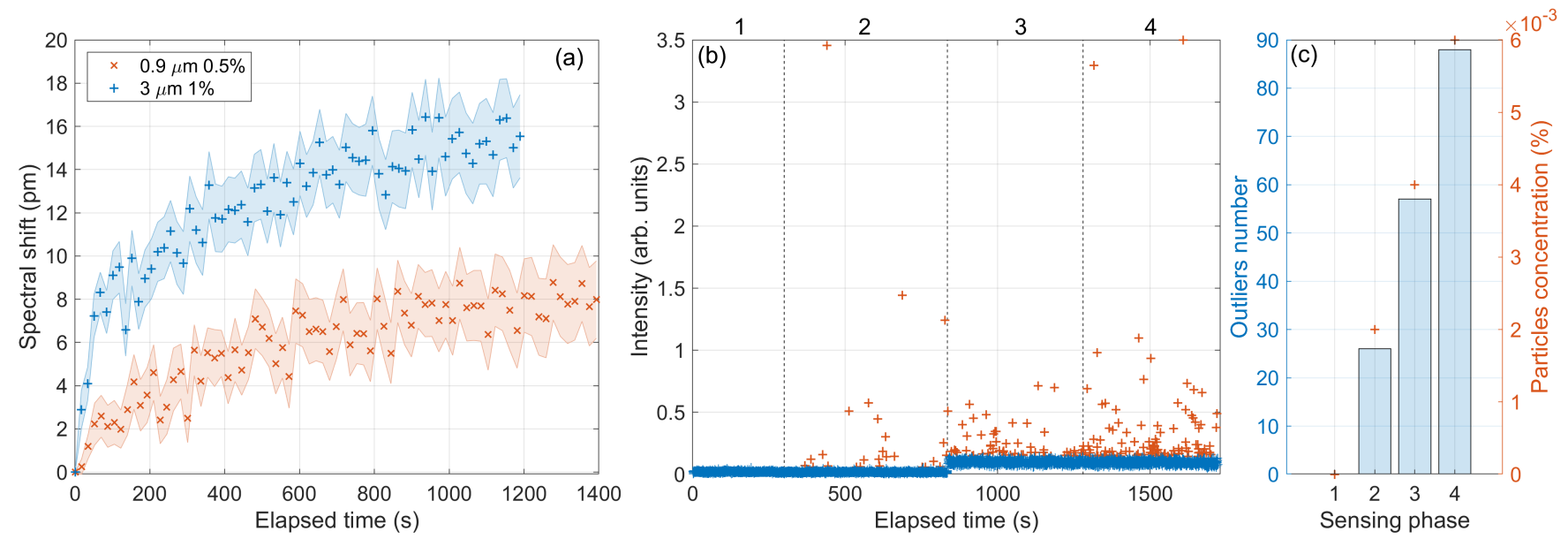

Fig. 7. PS microparticle detection with the WGM imaging sensor. (a) Generalized spectral shift for the wavelength sweeping interrogation (shaded area shows the 95\% confidence interval) [20] (@[2020] IEEE). (b) Signal variations for a selected WGM microresonator for the fixed frequency interrogation with respect to the following PS particle concentrations $0 \%(1), 2 \cdot 10^{-3} \%$ (2), $4 \cdot 10^{-3} \%$ (3), $6 \cdot 10^{-3} \%$ (4). (c) Statistics on the number of the detected outliers at the different sensing phases with respect to the particle concentration.

up to $\approx 2$ pm during $1200 \mathrm{~s}$. On the one hand, the behaviour of the spectral shift changes correlates well with the typical kinetics for small molecules adsorption. On the other hand, the obtained saturation values explicitly indicate the response on the bulk refractive index variation caused by the different concentrations of the dispersed particles. Here, the spectral shift values of $8 \mathrm{pm}$ for $0.5 \%$ concentration and of $16 \mathrm{pm}$ for $1 \%$ concentration correlate well with the numerical estimations (7 pm (TE) and $9 \mathrm{pm}(\mathrm{TM})$ for $0.5 \%$; $14 \mathrm{pm}$ (TE) and $17 \mathrm{pm}$ (TM) for 1\%) represented in Fig. 4b. Thus, the measured temporal dynamics in the experimental data is related to the mixing of the PS particles suspension with the previous solution in the fluidic cell (water) until the equilibrium is reached. This conclusion is also confirmed by the same time period required for both suspensions to reach saturation values. The discussed possibility for single microparticle detection has not been observed for the wavelength sweeping technique.

In order to analyse the performance of the fixed wavelength interrogation approach for the PS particles detection, the suspensions of $3 \mu \mathrm{m}$ microparticles dissolved in the deionized water at different final concentrations $\left(2 \cdot 10^{-3} \%, 4 \cdot 10^{-3} \%\right.$, $6 \cdot 10^{-3} \%$ ) have been prepared. The measured WGM data for a single microresonator for different sensing phases is represented in Fig. 7b. The results reveal slight variations of the median WGM signal between the different concentrations of PS microparticles that are defined by the bulk refractive index changes on the level below $10^{-5}$. At the same time, the registered signal contains outliers whose frequency and amount correlate well with the concentration of the PS particles in the sensed medium. The initial state (phase 1) where no particles are present in the medium contains no outliers whereas their number increases up to 26,57 , and 88 for $2 \cdot 10^{-3} \%, 4 \cdot 10^{-3} \%$, and $6 \cdot 10^{-3} \%$ concentration levels, correspondingly (Fig. 7c). A similar behaviour is observed for other microresonators as well where the amount of the appearing outliers directly depends on the particle concentration.

\section{DISCUSSION AND CONCLUSION}

The measured thermal sensitivity is comparable to the values reported previously for sensing configurations on single glass and silicon optical spherical microcavities [22], [23] with a benefit of the improved affordability in case of the discussed multiresonator imaging device. At the same time, the moderate sensitivity of the glass-based optical microcavities on the thermal variations is mainly limited by their relatively low thermal expansion coefficient. A common way to address this issue is to employ sensors out of materials demonstrating larger thermo-optic coefficients. For example, the PDMS and UV-curable photoresists allow the sensitivity enhancement by up to $\sim 10-100$ times. However, the atmospheric stability of the glass microresonators exceeds that one of the polymerbased cavities which is an essential benefit for the aerospacerelated sensing tasks. A trade-off solution for the sensing resolution enhancement and high sensor robustness may arise from the deposition of thin layers out of the materials with high thermo-optic coefficients on a stable glass resonator body. However, the resonator quality factor and thus the sensor detection limit might suffer from the insufficient accuracy of the sensing layer deposition. The next possible option may be the integration of the microresonators out of the materials with different thermo-optic coefficients realized in the form of a single multiresonator device. This approach is expected to be also effective for differentiation of the sensor responses on simultaneous external perturbations of different nature and will be addressed in the future studies.

The observed results on the particles detection clearly show the possibility for direct conversion of the spectral shift data collected with the wavelength sweeping technique into the mass concentration of the microparticles despite rather restricted separation between the microparticles of different size but equal mass concentration. At the same time, the fast response time of the fixed wavelength interrogation technique enables detectability of single microparticles where appearance and the amount of the outliers in the WGM signal are 
directly related to the presence of the particles. The complex nature of the WGM signal together with the concurrent impact of the external factors introduces, though, a rather high degree of randomness into the captured signal. The mentioned factors include properties of the sensed particle, its speed and instant position relative to the sensor surface. This point of transformation of the measured signal into the properties of the microparticles and corresponding formalization of the single microparticle detection principle will be addressed in the future studies.

The adhesive-layer based immobilization of the microcavities with respect to the coupling prism together with the sufficient stability of the adhesive layer in the ambient conditions allows to significantly reduce the impact of the vibrations and the airflow disturbances onto the coupling conditions. For the reported microcavities with Q-factors up to $10^{5}$, the instrument-caused uncertainties (laser wavelength and output power stability together with the detector noise) prevail. The repeatability of the wavelength scanning range is measured on the level of $1 \mathrm{pm}$ while utilizing the wavelength sweeping signal collection mechanism. The stability of the laser wavelength in the fixed wavelength illumination approach is measured to be below $0.3 \mathrm{pm}$ with relative variations of the output power at $10^{-4}$. Taking into account the measured sensitivity, the detection limit of the reported optical sensor configuration is expected to be on the level of $10^{-2}{ }^{\circ} \mathrm{C}$. The detection limit for the mass concentration for microparticles is close to the $0.05 \%$.

To conclude, we reported on a possible application of the WGM multiresonator imaging sensor for the aerial parameter detection. The unique sensor design, method for its characterization, signal collection and processing, together with both theoretical and experimental analysis of the sensing capabilities on example of the temperature and small particles sensing, have been discussed. We have demonstrated the sensing capabilities of the device for detection of the temperature variations in the range from $22.6^{\circ} \mathrm{C}$ to $26.1^{\circ} \mathrm{C}$ by analysing the resonant wavelength spectral shift with sensitivity of $8.6 \mathrm{pm} /{ }^{\circ} \mathrm{C}$. We have performed successful detection of the PM-like components in two different interrogation configurations (wavelength sweeping and spectrally unresolved). Good agreement of the experimental results on detection of $0.5 \%$ and $1 \%$ microparticles suspensions monitored with the wavelength sweeping technique together with the possibility for single particles detection in the fixed frequency illumination scheme have been demonstrated.

\section{REFERENCES}

[1] V. B. Braginsky, M. L. Gorodetsky, and V. S. Ilchenko, "Quality-factor and nonlinear properties of optical whispering-gallery modes," Physics Letters A, vol. 137, no. 7-8, pp. 393-397, 1989.

[2] K. J. Vahala, "Optical microcavities," Nature, vol. 424, no. 6950, pp. 839-846, 2003.

[3] M. R. Foreman, J. D. Swaim, and F. Vollmer, "Whispering gallery mode sensors," Advances in optics and photonics, vol. 7, no. 2, pp. 168-240, 2015.

[4] Y. Zhi, X.-C. Yu, Q. Gong, L. Yang, and Y.-F. Xiao, "Single nanoparticle detection using optical microcavities," Advanced materials (Deerfield Beach, Fla.), vol. 29, no. 12, 2017.
[5] T. J. Kippenberg, S. M. Spillane, D. K. Armani, and K. J. Vahala, "Fabrication and coupling to planar high-q silica disk microcavities," Applied Physics Letters, vol. 83, no. 4, pp. 797-799, 2003.

[6] G. Schweiger, R. Nett, and T. Weigel, "Microresonator array for highresolution spectroscopy," Optics Letters, vol. 32, no. 18, p. 2644, 2007.

[7] I. M. White, H. Zhu, J. D. Suter, N. M. Hanumegowda, H. Oveys, M. Zourob, and X. Fan, "Refractometric sensors for lab-on-a-chip based on optical ring resonators," IEEE Sensors Journal, vol. 7, no. 1, pp. 28 35, 2007.

[8] A. Chiasera, Y. Dumeige, P. Féron, M. Ferrari, Y. Jestin, G. Nunzi Conti, S. Pelli, S. Soria, and G. C. Righini, "Spherical whispering-gallery-mode microresonators," Laser \& Photonics Reviews, vol. 4, no. 3, pp. 457482, 2010.

[9] T. Wienhold, S. Kraemmer, S. F. Wondimu, T. Siegle, U. Bog, U. Weinzierl, S. Schmidt, H. Becker, H. Kalt, T. Mappes, S. Koeber, and C. Koos, "All-polymer photonic sensing platform based on whisperinggallery mode microgoblet lasers," Lab on a chip, vol. 15, no. 18, pp. 3800-3806, 2015.

[10] I. M. White and X. Fan, "On the performance quantification of resonant refractive index sensors," Optics express, vol. 16, no. 2, pp. 1020-1028, 2008.

[11] T. Reynolds, N. Riesen, A. Meldrum, X. Fan, J. M. M. Hall, T. M. Monro, and A. François, "Fluorescent and lasing whispering gallery mode microresonators for sensing applications," Laser \& Photonics Reviews, vol. 11, no. 2, p. 1600265, 2017.

[12] S. F. Wondimu, M. Hippler, C. Hussal, A. Hofmann, S. Krämmer, J. Lahann, H. Kalt, W. Freude, and C. Koos, "Robust label-free biosensing using microdisk laser arrays with on-chip references," Optics express, vol. 26, no. 3, pp. 3161-3173, 2018.

[13] Y.-Z. Yan, C.-L. Zou, S.-B. Yan, F.-W. Sun, Z. Ji, J. Liu, Y.-G. Zhang, L. Wang, C.-Y. Xue, W.-D. Zhang, Z.-F. Han, and J.-J. Xiong, "Packaged silica microsphere-taper coupling system for robust thermal sensing application," Optics express, vol. 19, no. 7, pp. 5753-5759, 2011.

[14] F. Monifi, S. K. Ozdemir, J. Friedlein, and L. Yang, "Encapsulation of a fiber taper coupled microtoroid resonator in a polymer matrix," IEEE Photonics Technology Letters, vol. 25, no. 15, pp. 1458-1461, 2013.

[15] X. Xu, W. Chen, G. Zhao, Y. Li, C. Lu, and L. Yang, "Wireless whispering-gallery-mode sensor for thermal sensing and aerial mapping," Light: Science \& Applications, vol. 7, p. 62, 2018.

[16] E. Krioukov, D. J. W. Klunder, A. Driessen, J. Greve, and C. Otto, "Sensor based on an integrated optical microcavity," Optics Letters, vol. 27, no. 7, pp. 512-514, 2002.

[17] S. Feng, T. Lei, H. Chen, H. Cai, X. Luo, and A. W. Poon, "Silicon photonics: From a microresonator perspective," Laser \& Photonics Reviews, vol. 6, no. 2, pp. 145-177, 2012.

[18] A. V. Saetchnikov, E. A. Tcherniavskaia, V. V. Skakun, V. A. Saetchnikov, and A. Ostendorf, "Reusable dispersed resonators-based biochemical sensor for parallel probing," IEEE Sensors Journal, vol. 19, no. 17, pp. 7644-7651, 2019.

[19] A. V. Saetchnikov, E. A. Tcherniavskaia, V. A. Saetchnikov, and A. Ostendorf, "Mapping of the detecting units of the resonator-based multiplexed sensor," in Optical Micro- and Nanometrology VII, C. Gorecki, A. K. Asundi, and W. Osten, Eds. SPIE, 2018, p. 33.

[20] A. Saetchnikov, E. Tcherniavskaia, V. Saetchnikov, and A. Ostendorf, "Application of dispersed microresonator based sensor for aerospacerelated tasks," in 2020 IEEE 7th International Workshop on Metrology for AeroSpace (MetroAeroSpace). IEEE, 2020, pp. 387-391.

[21] A. Saetchnikov, V. Skakun, V. Saetchnikov, E. Tcherniavskaia, and A. Ostendorf, "Quantification of whispering gallery mode spectrum variability in application to sensing nanobiophotonics," Journal of Nanophotonics, vol. 11, no. 04, p. 1, 2017.

[22] Q. Ma, T. Rossmann, and Z. Guo, "Temperature sensitivity of silica micro-resonators," Journal of Physics D: Applied Physics, vol. 41, no. 24 , p. 245111,2008

[23] J. Yu, E. Lewis, G. Brambilla, and P. Wang, "Temperature sensing performance of microsphere resonators," Sensors (Basel, Switzerland), vol. 18, no. 8, 2018. 